\title{
Trigeminal nerve and pathologies in magnetic resonance imaging - a pictorial review
}

\author{
Altan Gunes ${ }^{1 A, B, D, E, F}$, Elif Bulut ${ }^{2 B, E, F}$, Ayca Akgoz ${ }^{2 B, E, F}$, Burce Mocan ${ }^{2 A, B, E}$, Rahsan Gocmen ${ }^{2 B, E, F}$, Kader Karli Oguz ${ }^{2 A, B, E}$ \\ 'Department of Radiology, Ankara Children's Hematology and Oncology Training and Research Hospital, Diskapı, Ankara, Turkey \\ 2Department of Radiology, Hacettepe University Faculty of Medicine, Sihhiye, Ankara, Turkey
}

\begin{abstract}
A variety of conditions may affect the trigeminal nerve. Magnetic resonance imaging is the modality of choice when trigeminal nerve pathology is suspected, and this modality plays an essential role in detecting causes. This review illustrates some of the pathological conditions relevant to the trigeminal nerve in magnetic resonance imaging.
\end{abstract}

Key words: trigeminal nerve pain, magnetic resonance imaging.

\section{Introduction}

The trigeminal nerve $(\mathrm{TN})$ is the largest cranial nerve, which has a broad distribution territory [1]. It is a mixed sensitive-motor cranial nerve, receiving sensory afferent fibres from the face and mouth, and providing motor innervation to the muscles of mastication. A wide range of pathologies, including vascular, ischaemic, inflammatory, and neoplastic, can affect TN. Magnetic resonance imaging (MRI) is considered the primary method for evaluating patients with symptoms related to TN.

TN originates from the brainstem and follows a long and complex course towards its distribution territory. TN can be divided into four segments (brainstem, cisternal, Meckel's cave-cavernous sinus, extracranial). The nucleus of TN is found in the brainstem. Its roots exit from the pons, traverse the prepontine cistern, and enter Meckel's cave where the ganglion of TN lies and forms the peripheral branches (ophthalmic [V1], maxillary [V2], mandibular [V3]). V1 and V2 continue within the cavernous sinus and then $\mathrm{V} 1$ reaches the orbit via the superior orbital fissure and $\mathrm{V} 2$ reaches the pterygopalatine fossa via the foramen rotundum. V3 exits Meckel's cave, enters the foramen ovale, and joins with the motor root, which bypasses the TN gan- glion just below the foramen and reaches the infratemporal fossa $[1,2]$. The purpose of this review is to show MRI findings of the pathology of TN by using a segmental approach.

\section{Causative lesions of the brainstem}

The most common brainstem lesions causative for TN symptoms are multiple sclerosis, glioma, and infarction [3]. Demyelinating lesions of multiple sclerosis sometimes affect the pontine trigeminal pathways [4]. On T2-weighted and fluid attenuation inversion recovery (FLAIR) imaging, a high signal intensity ovoid lesion is seen in the area of the trigeminal nucleus. Similar additional lesions may be identified in the periventricular or juxtacortical/cortical white matter and the cerebellum, confirming dissemination in the space criteria of McDonald [5] (Figure 1).

Expansion of the brainstem by infiltration of the tumour can cause multiple cranial nerve palsies. The most common form of brainstem tumour is glioma, which mostly arises in the pons. Brainstem gliomas constitute $10-20 \%$ of all posterior fossa tumours in childhood but occur also less commonly in adults [6]. MRI is the most accurate way to diagnose and to assess the extent of the tumour [6] (Figure 2).

\footnotetext{
Correspondence address:

Altan Gunes, MD, Department of Radiology, Ankara Children's Hematology and Oncology Training and Research Hospital, Diskapı, 06130, Ankara, Turkey,

phone: +90-0312-596-96-00, fax: +90-0312-347-23-30, e-mail: draltangunes@gmail.com
}

Authors' contribution:

A Study design · B Data collection · C Statistical analysis · D Data interpretation · E Manuscript preparation · F Literature search · G Funds collection 


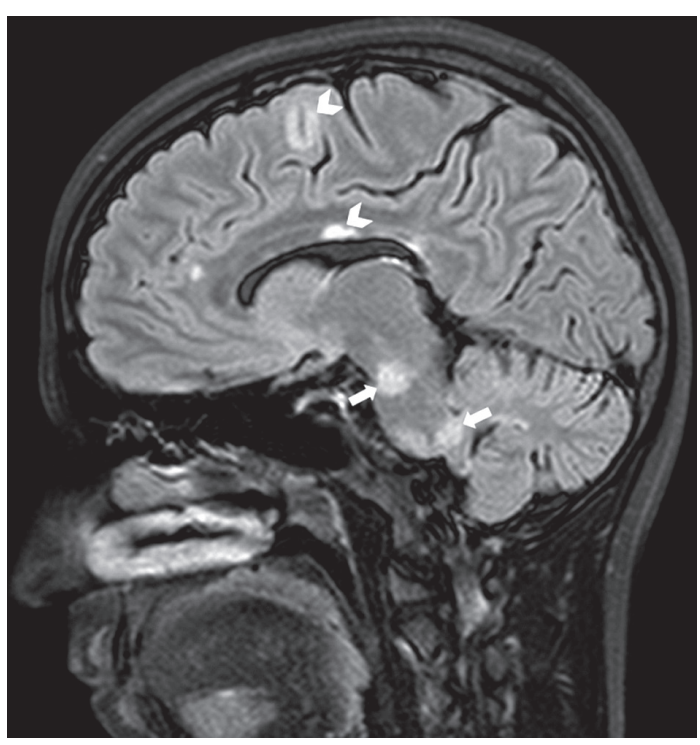

Figure 1. A 36-year-old woman with diagnosis of multiple sclerosis. Sagittal FLAIR image reveals demyelinating lesions in the area of the trigeminal nucleus (arrows) and plaques at the callososeptal interface and the juxtacortical white matter (arrowheads)

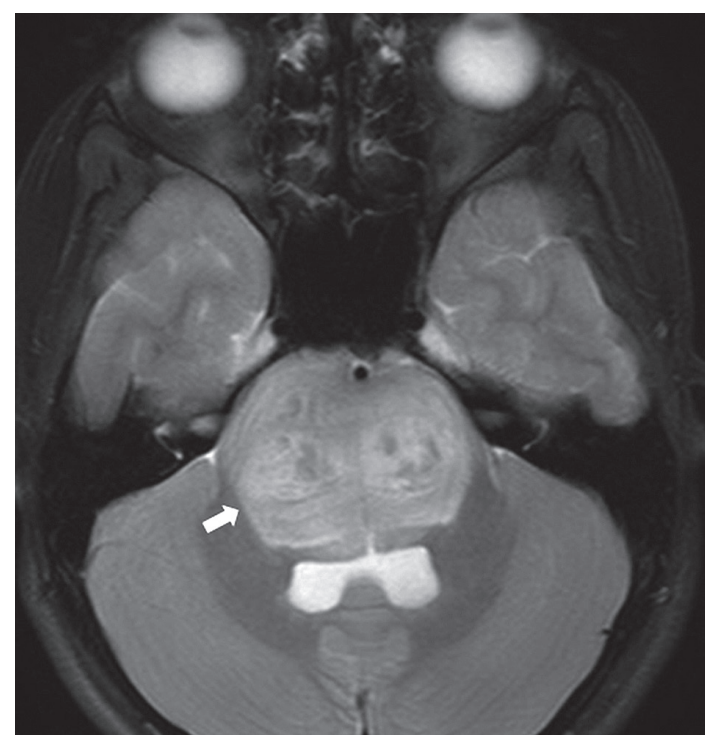

Figure 2. An eight-year-old girl with brainstem glioma. Axial fat-suppressed, T2-weighted image shows a hyperintense mass expanding and infiltrating the pons (arrow)
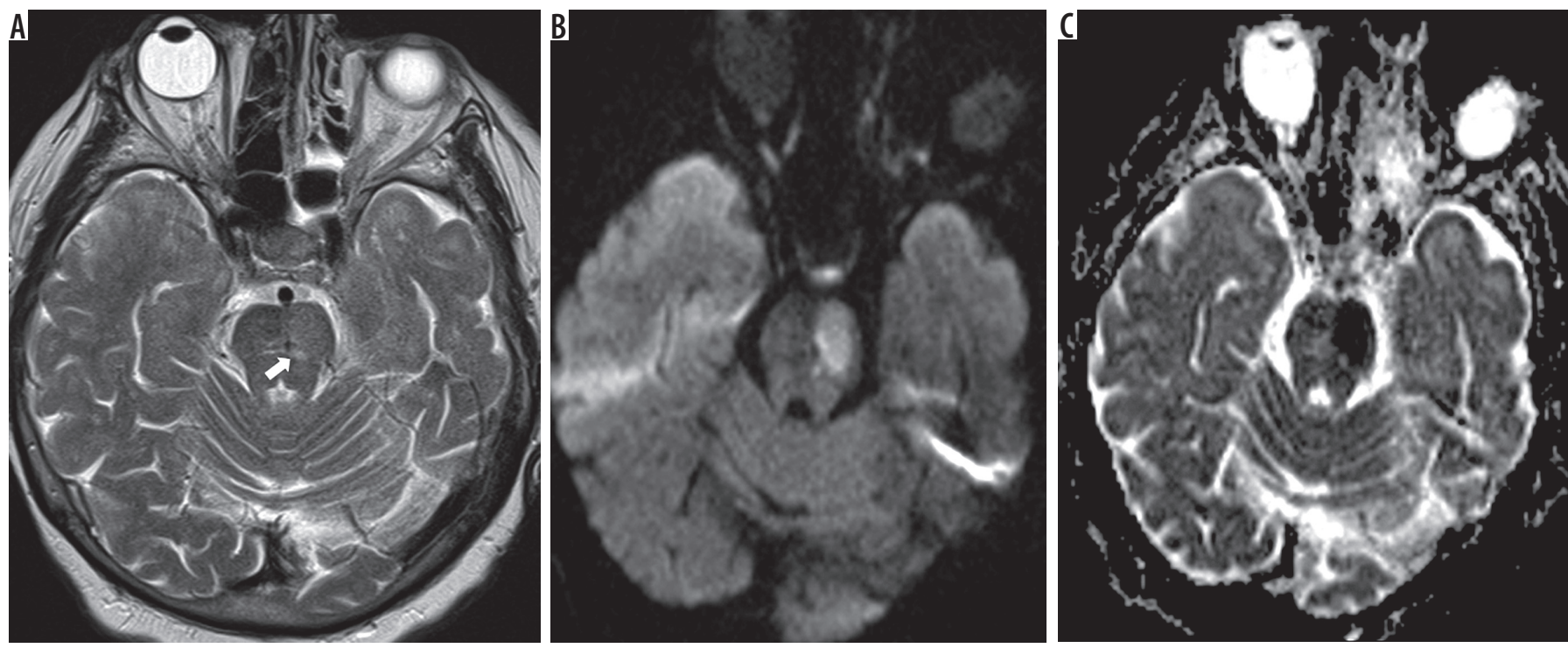

Figure 3. A 56-year-old woman with brainstem infarction. A) Localised mild hyperintensity by area of infarction (arrow) on the left paramedian upper pons is seen on T2-weighted image. B) The DW image and ADC map (C) clearly show acute brainstem infarction by restricted diffusion

The infarction of the brainstem often involves impairment both of the cranial nuclei and the long tracts and can cause nerve palsies. Most of the brainstem infarctions are ischaemic and occur secondarily to occlusion of small perforating arteries of the posterior circulation. Diffusion-weighted imaging (DWI) is useful to look for ischemic lesions [7] (Figure 3).

\section{Causative lesions of the cistern}

TN symptoms can be caused by vascular and neoplastic lesions within the cistern [3]. Neurovascular compression is defined as abnormal contact between the vessel and the root entry zone, which is situated adjacent to the pons. This compression is the most common cause of trigeminal neuralgia [3]. Tortuous branches of posterior circulation vessels, especially the superior cerebellar artery, may compress TN and cause focal loss of myelin [8,9]. Other vascular lesions (aneurysms, arteriovenous malformations, dural arteriovenous fistula, and ectasias) may also result in neurovascular compression. This compression is best demonstrated on 3D high-resolution steady-state free precession (SSFP) sequences and 3D time-of-flight (TOF) angiography (Figure 4).

Schwannomas are the most common primary tumour of TN. Their localisations often determine the symptoms 

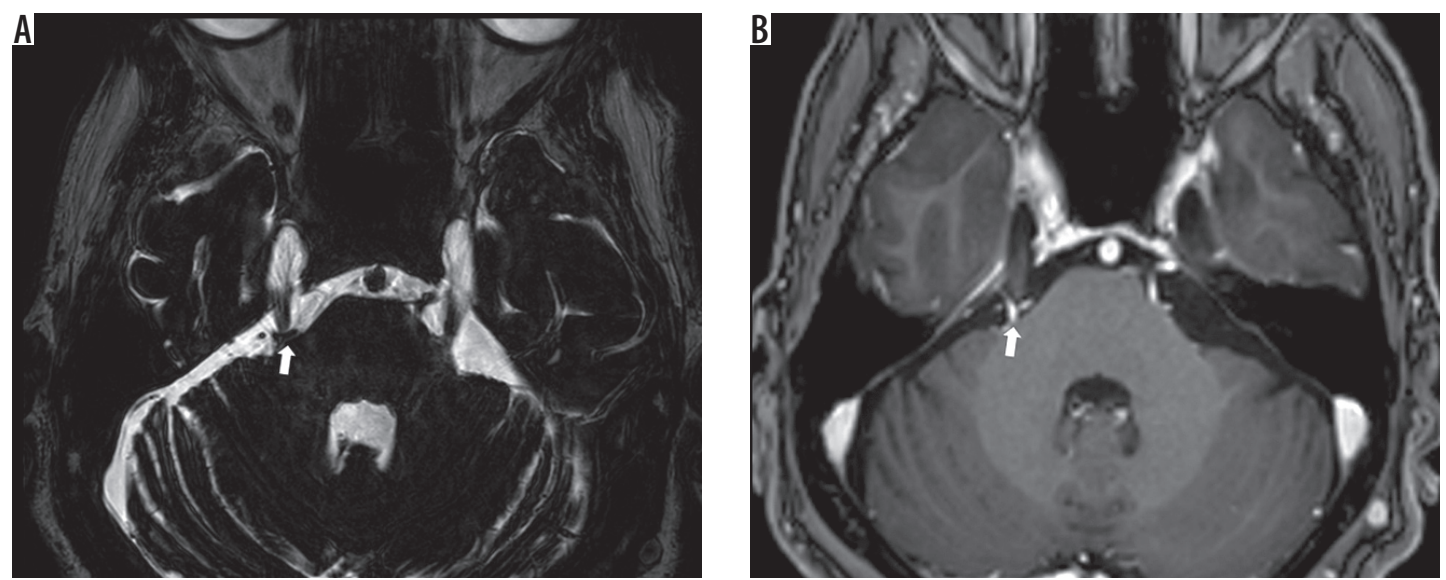

Figure 4. A 60-year-old woman with right trigeminal neuralgia. On axial DRIVE image (A), a venous structure (arrow) is seen indenting the right fifth nerve. Axial post-contrast 3D MP-RAGE image (B) shows neurovascular compression of the trigeminal nerve (arrow)
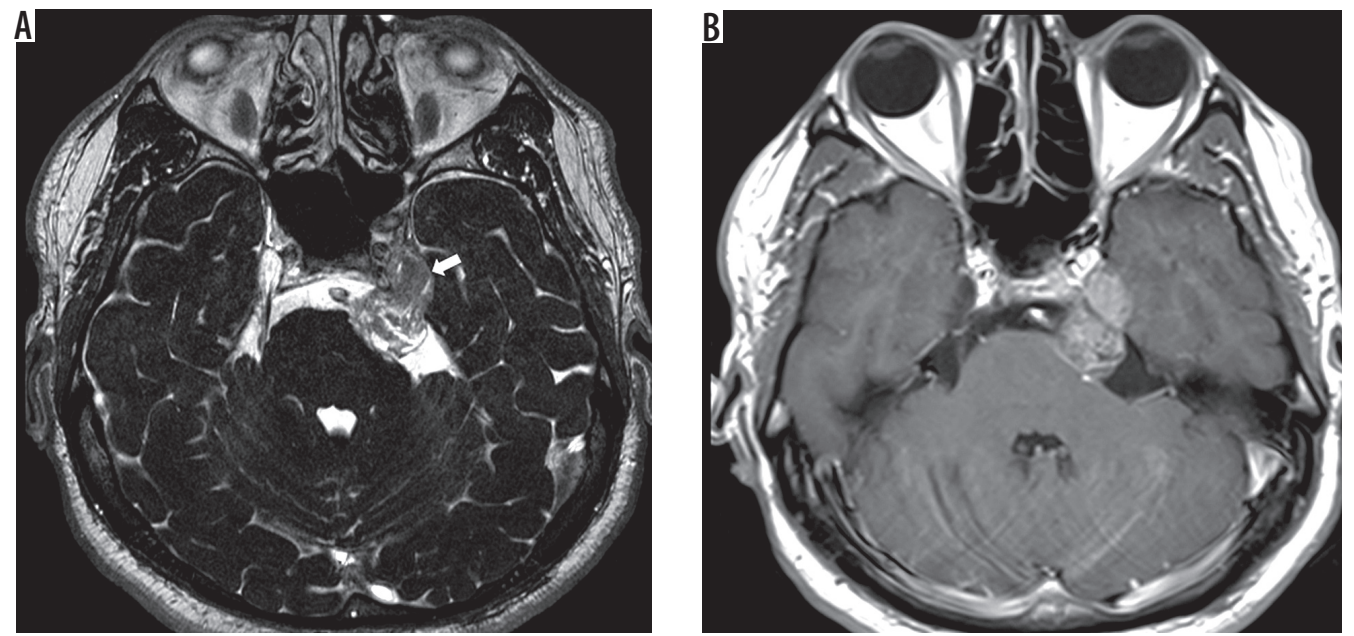

Figure 5. A 48-year-old man with trigeminal schwannoma. Axial DRIVE image (A) shows a dumbbell-shaped heterogeneous mass (arrow) crossing the prepontine cistern and Meckel's cave along course of the left trigeminal nerve. Axial post-contrast T1-weighted image (B) shows heterogeneous enhancement of the lesion

with which the patient presents. They may arise in any segment of TN and usually show homogenous enhancement (Figure 5). The large schwannomas can have inhomogeneous signal intensity due to haemorrhage and cyst formation (Figure 6).

TN may also be affected by lesions such as meningiomas and epidermoid cysts [10]. Meningiomas typically appear as extra-axial masses with a broad dural base and show intense homogenous enhancement with thickened adjacent dura [11] (Figure 7). An epidermoid cyst is a benign congenital lesion of ectodermal origin, the characteristic finding of which is a very bright signal on DWI [12] (Figure 8).

\section{The causative lesions of the Meckel's cave and cavernous sinus}

Meckel's cave or cavernous sinus may be involved by vascular, neoplastic, and inflammatory lesions. Intracavernous carotid aneurysms and carotid cavernous fistula

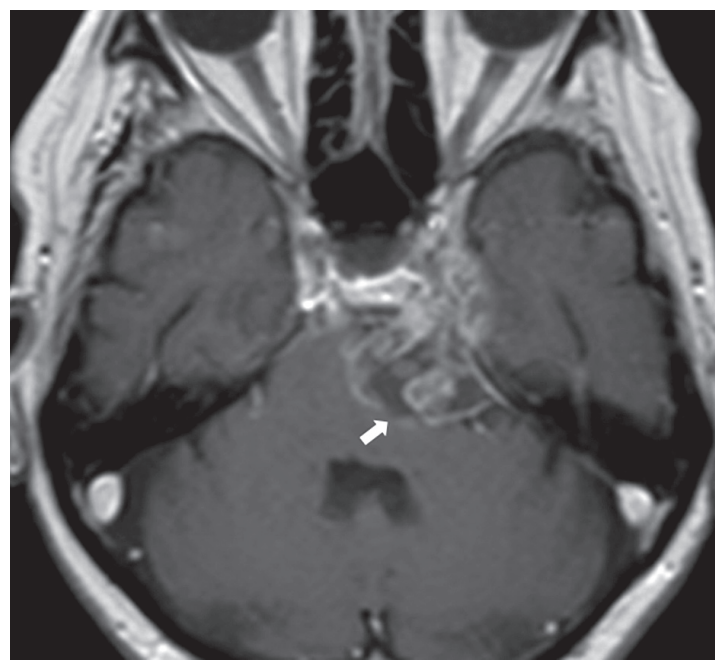

Figure 6. A 36-year-old woman with trigeminal schwannoma. Axial post-contrast T1-weighted image shows heterogenous enhancing cystic and solid mass along the left trigeminal nerve (arrow) 

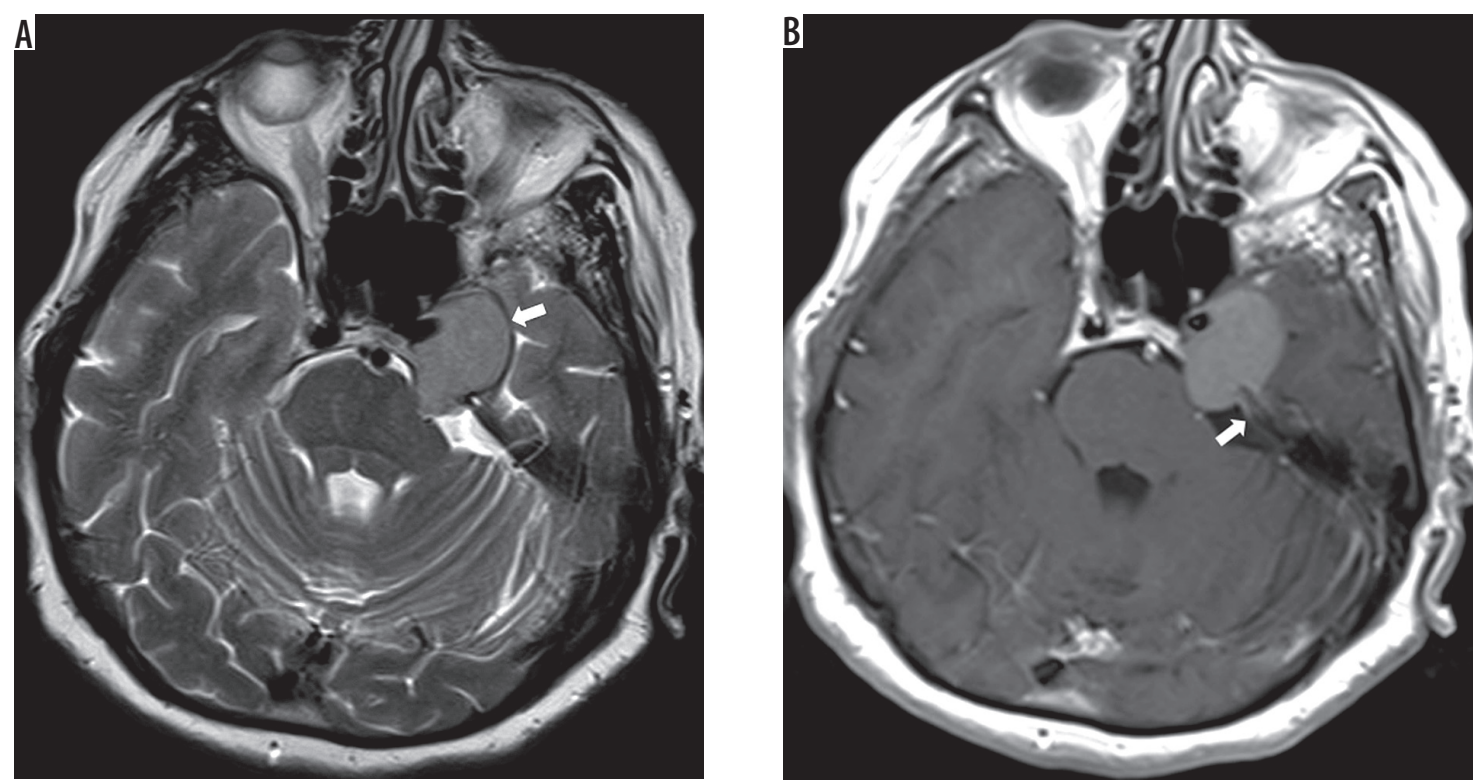

Figure 7. A 58-year-old woman referred for left trigeminal neuralgia. Axial T2-weighted image (A) shows meningioma involving Meckel's cave, cavernous sinus, and prepontine cistern (arrow). Axial post-contrast T1-weighted image (B) shows homogeneous enhancement of the lesion and dural tail (arrow)
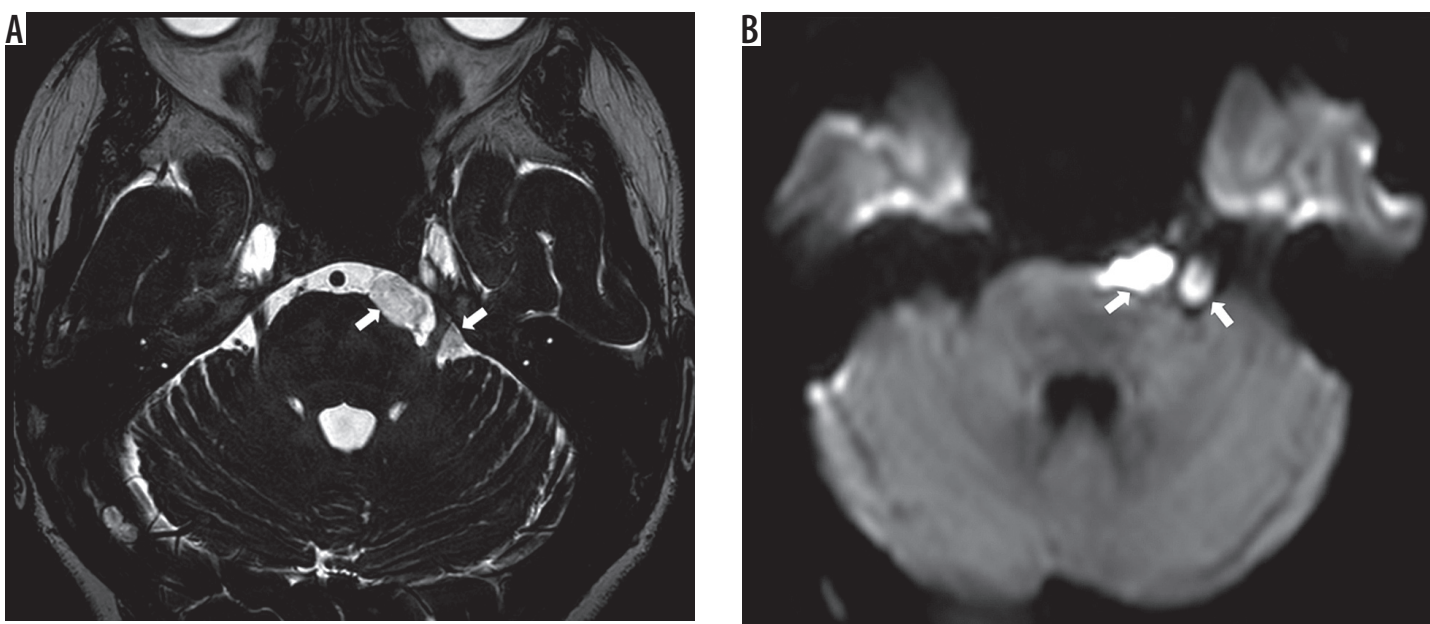

Figure 8. A 46-year-old man referred for left trigeminal neuralgia. Axial DRIVE image (A) demonstrates an epidermoid cyst (arrows) in the left prepontine cistern. An epidermoid cyst appears bright on the DW image (B)

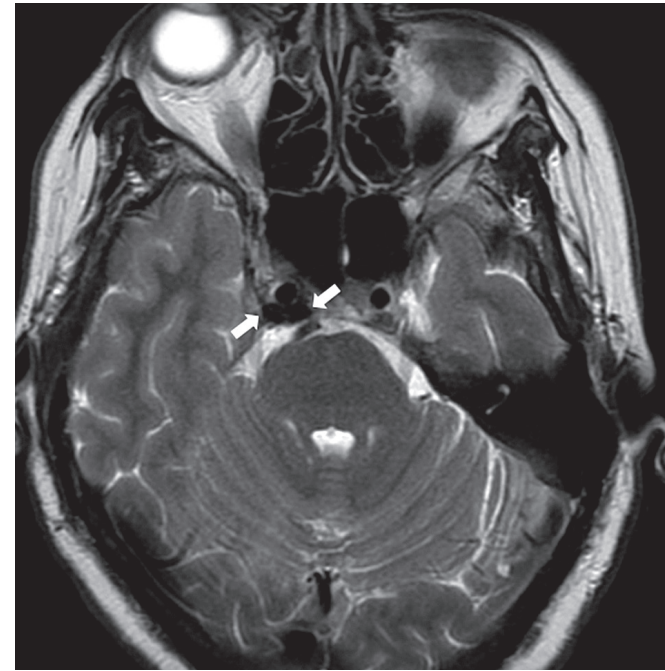

Figure 9. A 24-year-old woman with a diagnosis of carotid cavernous fistula. Axial T2-weighted image shows multiple signal void structures, indicating high flow in the right cavernous sinus (arrows)

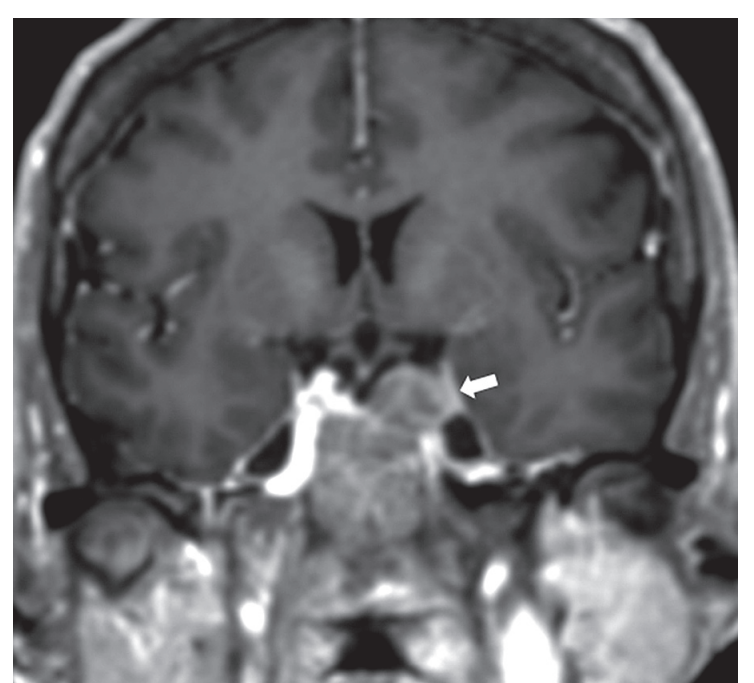

Figure 10. A 44-year-old woman with pituitary adenoma. Coronal post- contrast T1-weighted image shows invasion of enhancing pituitary mass into the left cavernous sinus, displacing the cavernous internal carotid artery (arrow) 
may compress the cavernous sinus and may be associated symptoms of TN $[8,13]$ (Figure 9). Also, some pituitary adenoma can infiltrate the cavernous sinus (Figure 10). MR imaging and TOF angiography are the most accurate ways to assess the extent of adenoma and to show the vascular anatomy and abnormality.

In inflammatory and infectious disease, such as Gradenigo's syndrome, the abducens nerve as well as the Gasserian ganglion may become involved [14]. The abnormal contrast enhancement of meninges, nerves, and cavernous sinus thrombosis can be seen on MR imaging (Figures 11 and 12).

Metastatic involvement of TN may be seen as direct infiltration or secondary to haematogenous-leptomeningeal dissemination (Figures 13-17). 3D contrast-enhanced magnetisation-prepared rapid-acquisition gradient-echo (MP-RAGE) sequence is very useful for demonstration of these lesions (Figure 18).
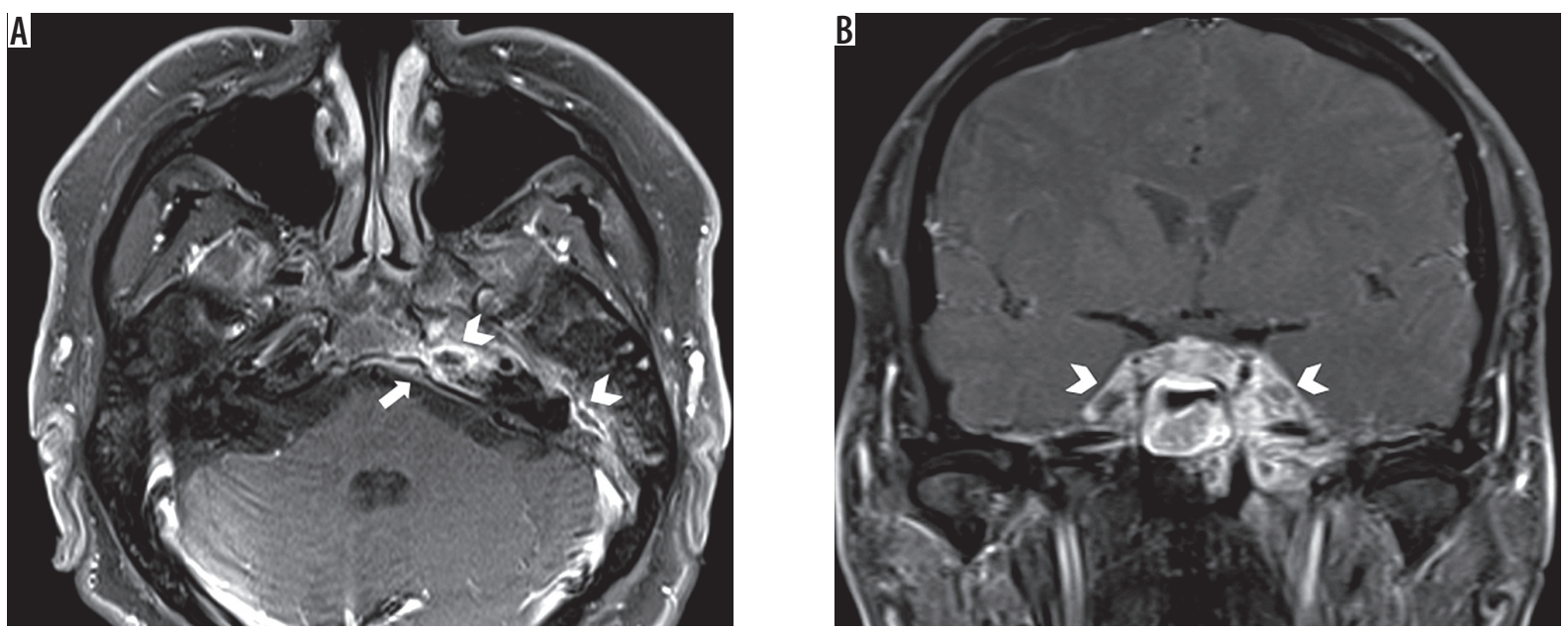

Figure 11. A 37-year-old man with petrous apicitis. Axial post-contrast T1-weighted image (A) with fat suppression shows diffuse enhancement throughout the left mastoid and middle ear petrous apex (arrowheads). There is also dural enhancement along the posterior margin of the petrous apex (arrow). Coronal post-contrast T1-weighted image (B) shows inflammation with extension into the bilateral cavernous sinus and Meckel's cave (arrowheads)

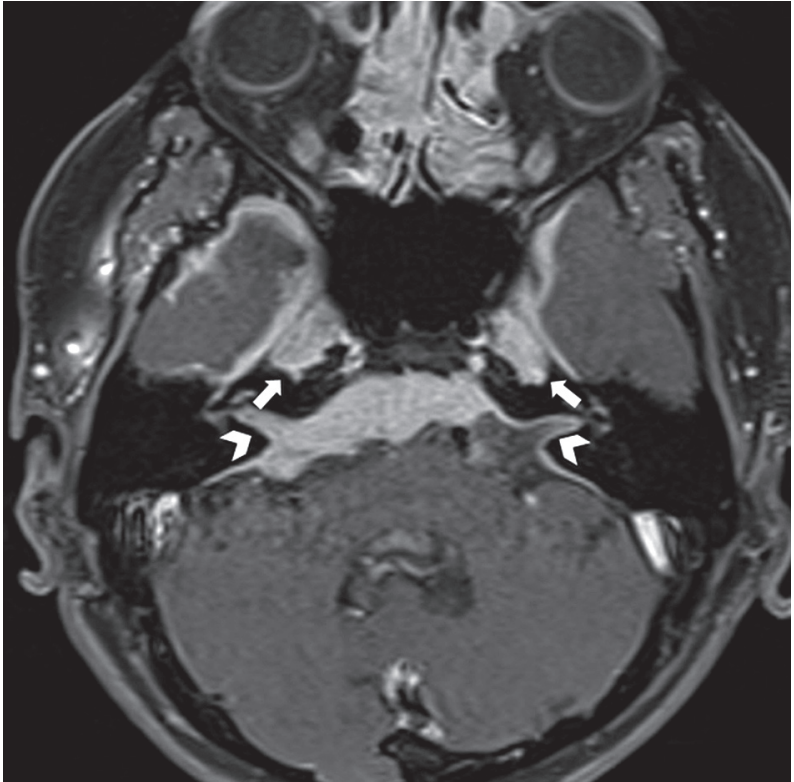

Figure 12. A 49-year-old man with Wegener's granulomatosis. Axial postcontrast T1-weighted image with fat suppression shows the diffuse dural thickening and enhancement with extension into the bilateral internal acoustic channel (arrowheads) and Meckel's cave (arrows)
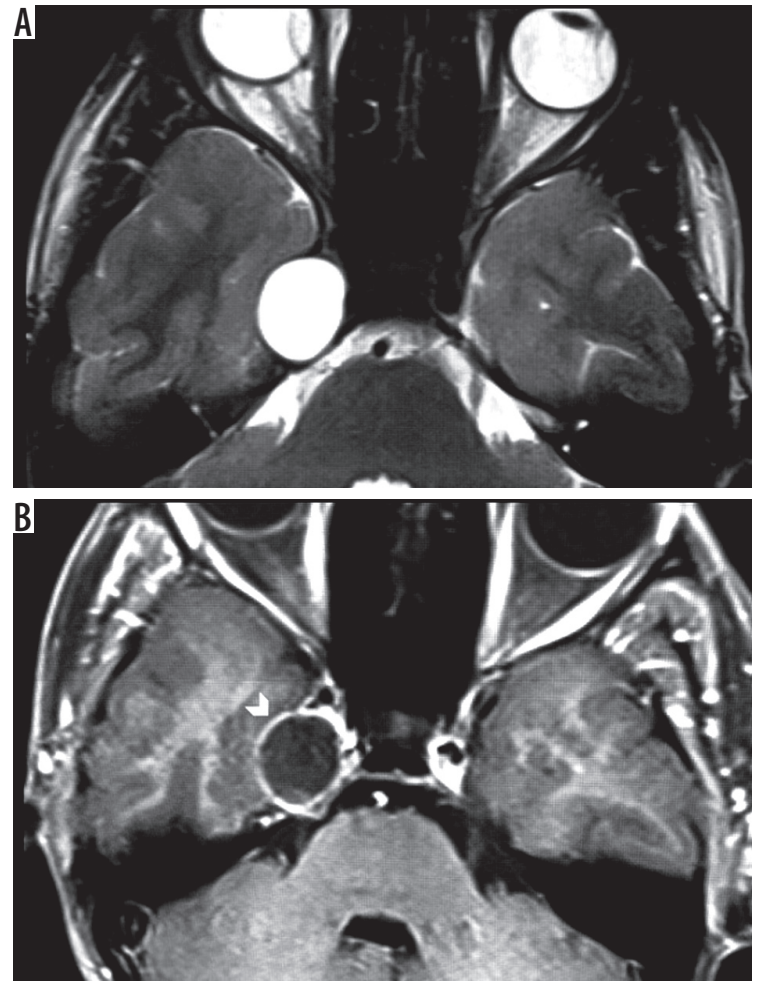

Figure 13. A 56-year-old woman with known diagnosis of squamous cell carcinoma of the skin, referred for right trigeminal neuralgia. Axial T2-weighted image (A) demonstrates cystic lesion in the right Meckel's cave. Axial post-contrast T1-weighted image (B) shows peripheral contrast enhancement of the metastatic lesion (arrowhead) 


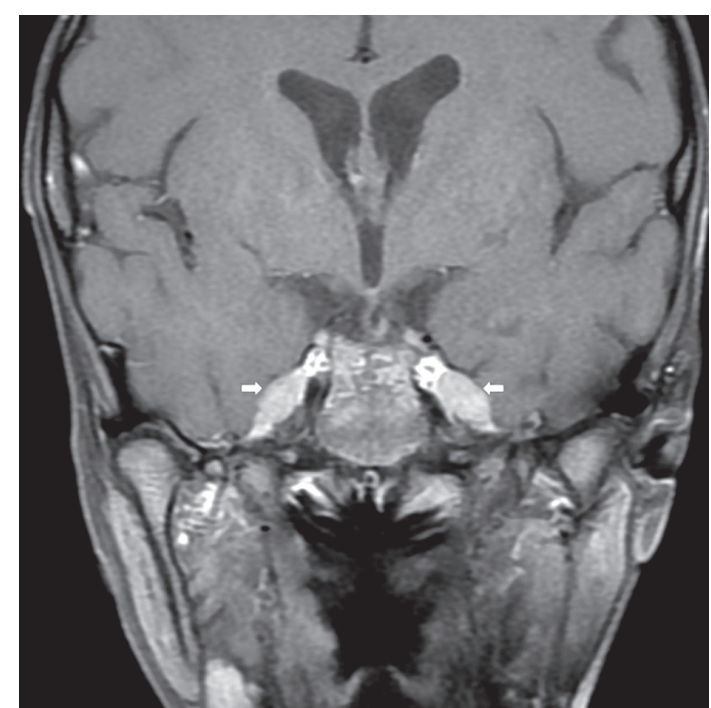

Figure 14. A 12-year-old man with known diagnosis of Burkitt lymphoma. Coronal post-contrast T1-weighted image shows diffuse enhancement throughout the cisternal and Meckel's cave segments of the trigeminal nerve (arrows)

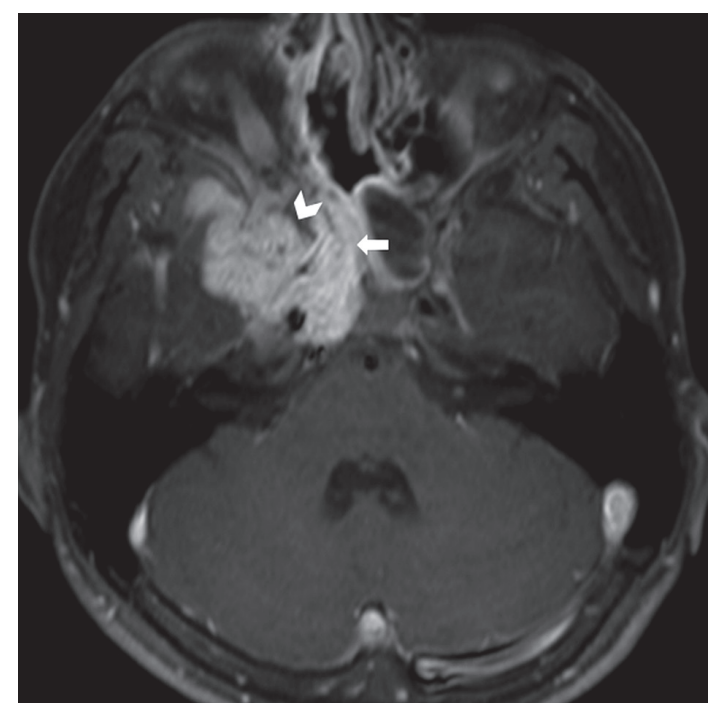

Figure 16. A 13-year-old man with juvenile nasopharyngeal angiofibroma. Axial post-contrast T1-weighted image with fat suppression demonstrates marked homogeneous enhancement of the lesion (arrow) with extension medially into the sphenoid sinus (arrow) and laterally into the pterygopalatine fossa and infratemporal fossa (arrowhead)

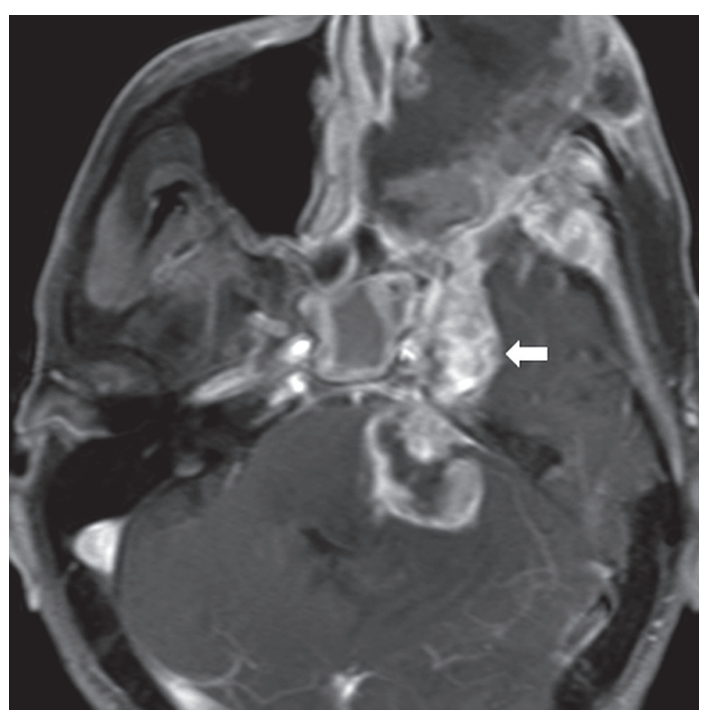

Figure 15. A 58-year-old man with squamous cell carcinoma of the skin. Axial post-contrast T1-weighted image with fat suppression shows heterogenous enhancing cystic and solid mass along the trigeminal nerve and its branches (arrow)

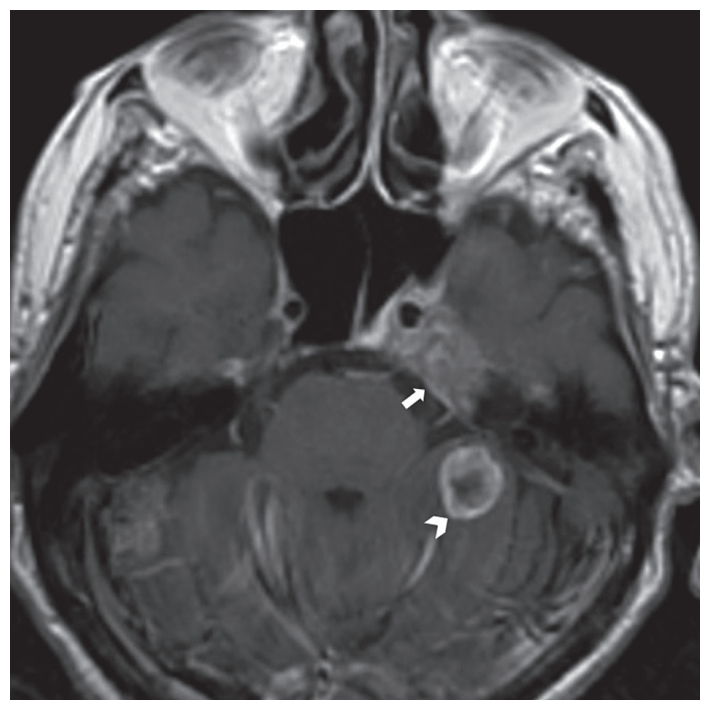

Figure 17. A 65-year-old man with known diagnosis of bladder cancer. On axial post-contrast T1-weighted image, metastases in the left Meckel's cave (arrow) and the left cerebellar hemisphere (arrowhead) are seen

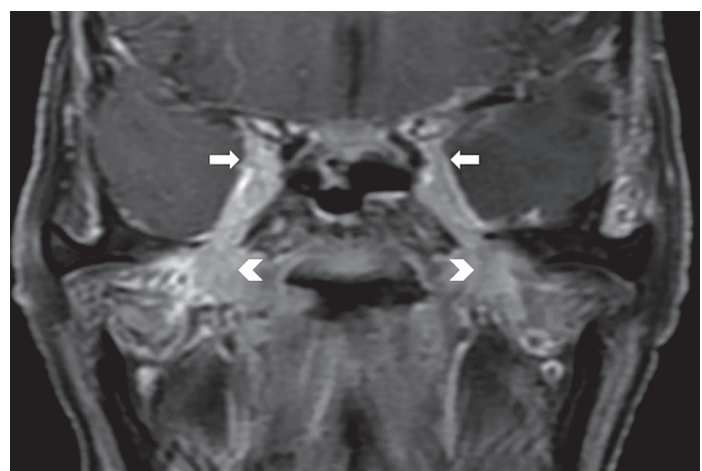

Figure 18. A 64-year-old man with adenoid cystic carcinoma of the skin. Coronal post-contrast T1-weighted image with fat suppression shows enhancement and irregular thickening of the bilateral mandibular branch of the trigeminal nerve and widening of the foramen ovale (arrowheads). Abnormal enhancement seems to extend into the bilateral Meckel's cave (arrows) 


\section{Causative lesions of the extracranial portion}

Extracranial causes of TN symptoms include neoplastic and fibro-osseous lesions. The branches of TN are most commonly involved in perineural spread of head and neck malignancies such as adenoid cystic carcinoma and squamous cell carcinoma [8]. On imaging, enhancement and irregular thickening of the nerve, widening of the neural foramen, and atrophy of the supplied muscles can be seen [15] (Figure 19).

The fibro-osseous lesions may affect the skull-base neural foramina and cause pressure or infiltration of TN

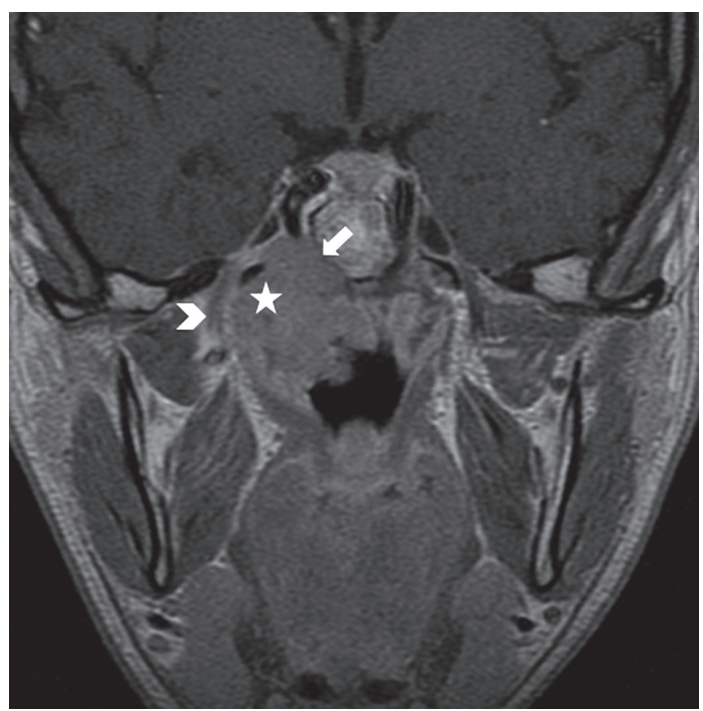

Figure 19. A 39-year-old man with nasopharyngeal carcinoma with perineural tumour spread. Coronal post-contrast T1-weighted image shows marked homogeneous enhancement of the lesion with extension superiorly into the foramen lacerum (asterisk) and cavernous sinus (arrow). Also note the abnormal thickening and enhancement of the right mandibular branch of the trigeminal nerve (arrowhead)

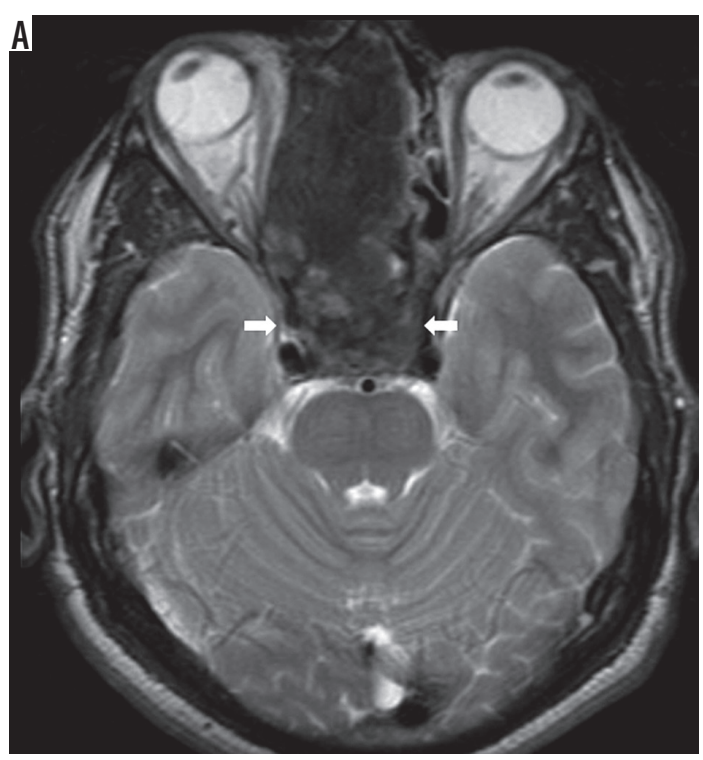

branches. Chordoma, chondromatous lesions, and metastases are common among these lesions (Figures 20 and 21).

\section{MRI of the trigeminal nerve pathologies}

Routine brain MR imaging protocols (T1-weighted spin echo, T2-weighted turbo spin echo, FLAIR, DWI, susceptibility-weighted imaging) help to exclude and evaluate other causes that could mimic the symptoms. A protocol should include 3D TOF angiography, and 3D SSFP and 3D MP-RAGE sequences before and after contrast injection. 3D SSFP sequences provide superior delineation of

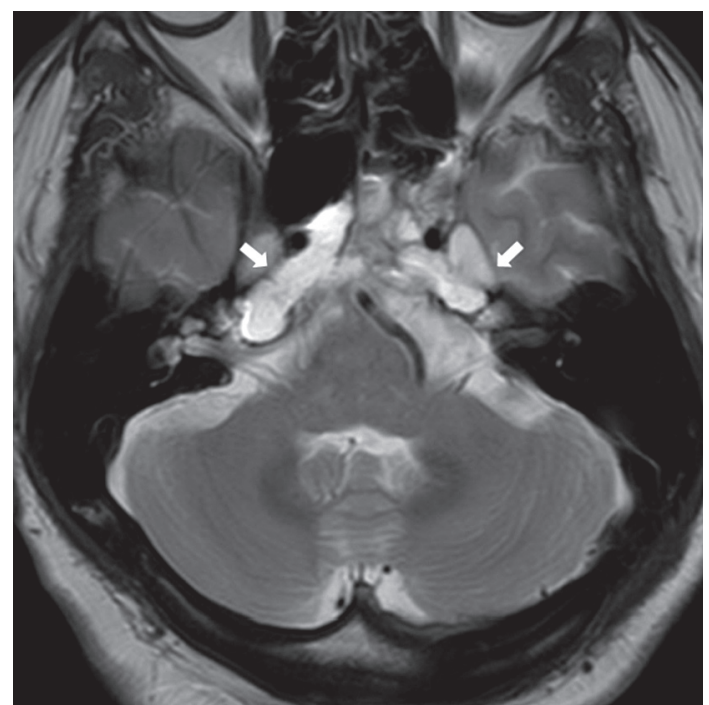

Figure 20. A 45-year-old man with chordoma. Axial T2-weighted image shows a clival chordoma with extension into the bilateral cavernous sinus and the right Meckel's cave (arrows)

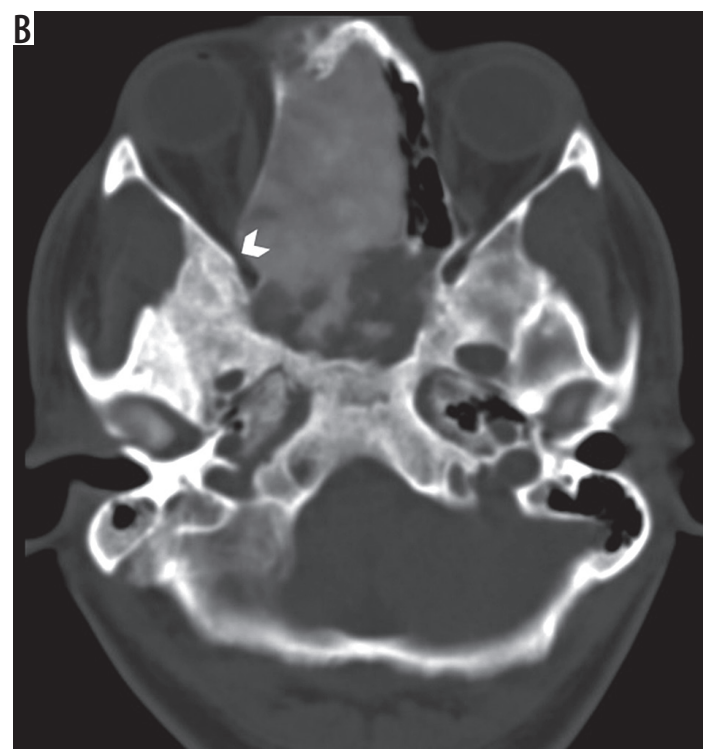

Figure 21. A 31-year-old man with fibrous dysplasia of the nasal cavity region. Lesion shows a heterogeneous low signal intensity on axial T2-weighted image (A) and significant narrowing of Meckel's caves (arrows). Axial bone-window computed tomography image (B) demonstrates ground-glass lesion that expands the nasal cavity and narrows the right orbital fissure (arrowhead) 
vascular structures and nerves in the cerebrospinal fluid spaces (Figures 4, 5 and 8). 3D TOF and MP-RAGE sequences are very helpful for demonstration of the neurovascular contact and contrast enhancing lesions along the course of the TN $[9,11]$ (Figures 4 and 12).

\section{Conclusions}

MR imaging has increased the role of the diagnosis of TN disorders with the implementation of high-resolution 3D imaging sequences. Knowledge of TN anatomy is essential for correct interpretation of the images and accurate diagnosis.

\section{Conflict of interest}

The authors report no conflict of interest.

\section{References}

1. Laine FJ, Smoker W. Anatomy of the cranial nerves. Neuroimag Clin N Am 1998; 8: 69-100.

2. Williams LS, Schmalfuss IM, Sistrom CL, et al. MR imaging of the trigeminal ganglion, nerve, and the perineural vascular plexus: normal appearance and variants with correlation to cadaver specimens. Am J Neuroradiol 2003; 24: 1317-1323.

3. Kamel $\mathrm{H}$, Toland J. Trigeminal nerve anatomy: illustrated using examples of abnormalities. Am J Roentgenol 2001; 176: 247-251.

4. Majoie C, Hulsmans F, Castelijns JA, et al. Symptoms and signs related to the trigeminal nerve: diagnostic yield of MR imaging. Radiology 1998; 209: 557-562.

5. Polman CH, Reingold SC, Banwell B, et al. Diagnostic criteria for multiple sclerosis: 2010 revisions to the McDonald criteria. Ann Neurol 2011; 69: 292-302.

6. Packer RJ, Nicholson HS, Vezina LG, Johnson DL. Brainstem Gliomas. In: Berger MS (ed.). Pediatric Neuro-oncology. Vol 3. Saunders, Philadelphia 1992; 863-879.

7. Valls-Solé J, Vila N, Obach V, et al. Brain stem reflexes in patients with Wallenberg's syndrome: correlation with clinical and magnetic resonance imaging (MRI) findings. Muscle Nerve 1996; 19: 1093 1099.
8. Borges A, Casselman J. Imaging the trigeminal nerve. Eur J Radiol 2010; 74: 323-340.

9. Lutz J, Linn J, Mehrkens JH, et al. Trigeminal neuralgia due to neurovascular compression: high-spatial-resolution diffusion-tensor imaging reveals microstructural neural changes. Radiology 2011; 258: 524-530.

10. Majoie C, Verbeeten Jr B, Dol JA, Peeters F. Trigeminal neuropathy: evaluation with MR imaging. Radiographics 1995; 15: 795-811.

11. Helie O, Soulie D, Sarrazin J, et al. Magnetic resonance imaging and meningiomas of the posterior cerebral fossa. 31 cases. J Neuroradiology 1995; 22: 252-270.

12. Fitt AW, Pigott TJ, Marks PV. Undiagnosed epidermoid cyst presenting as trigeminal neuralgia: a need for MRI. Brit J Neurosurg 1984; 8: 101-103.

13. Gonella MC, Fischbein NJ, So YT. Disorders of the trigeminal system. Semin Neurol 2009; 29: 36-44.

14 Lee JH, Lee HK, Park JK, et al. Cavernous sinus syndrome: clinical features and differential diagnosis with MR imaging. Am J Roentgenol 2003; 181: 583-590.

15. De Marco JK, Hesselink JR. Trigeminal neuropathy. Neuroimag Clin N Am 1993; 3: 105-128. 